\title{
Incontinentia pigmenti: genodermatosis multisistémica
}

\author{
Adrián Martínez-Gayosso y María T. García-Romero* \\ Servicio de Dermatología, Instituto Nacional de Pediatría, Ciudad de México, México
}

\section{Resumen}

La incontinentia pigmenti es una genodermatosis ligada al cromosoma $X$, generalmente letal en los hombres. Está causada por una mutación con pérdida de función en el gen IKBKG (inhibitor of kappa polypeptide gene enhancer in B cells kinase gamma), que impide que la proteína $N F \kappa \beta$ (nuclear factor kappa-light-chain-enhancer of activated B cells) migre al núcleo y comience la transcripción de factores que amplifican la respuesta inmunitaria y previenen la apoptosis. Por tanto, las células mutantes se vuelven vulnerables a la apoptosis cuando son expuestas a citocinas y provocan vaso-oclusión e isquemia de tejidos como la piel, el sistema nervioso central y la retina. Las lesiones dermatológicas son características; se distribuyen a lo largo de las líneas de Blaschko, las cuales siguen el patrón de migración de las células de la piel en la embriogénesis, y ocurren en el $100 \%$ de los pacientes. Las manifestaciones cutáneas aparecen en una secuencia de cuatro fases que inicia desde el nacimiento: vesicular, verrucosa, hiperpigmentada e hipopigmentada. Estas lesiones son relevantes, puesto que orientan al clínico hacia el diagnóstico. Además, se acompañan de anomalías neurológicas, como crisis convulsivas, y múltiples manifestaciones oftalmológicas, como el desprendimiento de la retina. Los pacientes con incontinentia pigmenti, pero sin compromiso oftalmológico o neurológico clínicamente significativo, tienen un pronóstico bueno y una esperanza de vida normal. Las anomalías que se presentan son permanentes, lo que puede generar preocupación en los pacientes.

Palabras clave: Incontinentia pigmenti. Trastornos pigmentarios. Enfermedad genética.

\section{Incontinentia pigmenti: multisistemic genodermatosis}

\begin{abstract}
Incontinentia pigmenti is an X-linked genodermatosis generally lethal in males; thus, it presents almost exclusively in females. It is caused by a loss-of-function mutation in the IKBKG (inhibitor of kappa polypeptide gene enhancer in $B$ cells, kinase gamma) gene that prevents the $N F_{\kappa} \beta$ (nuclear factor kappa-light-chain-enhancer of activated B cells) protein from migrating to the nucleus to begin the transcription of factors that amplify the immune response and prevent apoptosis. Consequently, mutant cells become vulnerable to apoptosis when exposed to cytokines and, in turn, lead to vaso-occlusion and ischemia of tissues, such as the skin, the central nervous system and the retina. Dermatological lesions are characteristic and occur in $100 \%$ of patients; they are distributed along Blaschko lines, which follow the pattern of migration of skin cells in embryogenesis. The cutaneous manifestations follow a sequence of four phases since birth: vesicular, verrucous, hyperpigmented and hypopigmented. These lesions are relevant for the disease because they guide the clinician towards the diagnosis. Additionally, they are accompanied by neurological abnormalities, such as seizures, and multiple ophthalmological manifes-
\end{abstract}

Correspondencia:

*María T. García Romero

E-mail: teregarro@gmail.com
Fecha de recepción: 24-10-2019

Fecha de aceptación: 27-11-2019

DOI: 10.24875/BMHIM.19000173
Disponible en internet: 23-04-2020

Bol Med Hosp Infant Mex. 2020;77(3):112-118

www.bmhim.com 1665-1146/@ 2019 Hospital Infantil de México Federico Gómez. Publicado por Permanyer. Este es un artículo open access bajo la licencia CC BY-NC-ND (http://creativecommons.org/licenses/by-nc-nd/4.0/). 
tations, such as retinal detachment. Incontinentia pigmenti patients with no clinically significant ophthalmic or neurological compromise have a good prognosis and a normal life expectancy. The abnormalities present are permanent, which can be a cause of concern for the patients.

Key words: Incontinentia pigmenti. Pigmentary disorders. Genetic diseases.

\section{Introducción}

La incontinentia pigmenti (IP), también llamada síndrome de Bloch Sulzberger, es una genodermatosis de herencia dominante ligada al cromosoma X, causada por una mutación con pérdida de función en el gen IKBKG (inhibitor of kappa polypeptide gene enhancer in $B$ cells kinase gamma), antes llamado NEMO (nuclear factor-kappa $B$ essential modulator) 1 . La IP es letal en el sexo masculino, por lo que, generalmente, se presenta de forma casi exclusiva en las mujeres, en quienes la presencia de un doble cromosoma $\mathrm{X}$ permite la inactivación constitutiva del alelo mutado, provocando el silenciamiento de estos genes. La inactivación no es completa al $100 \%$; por lo tanto, estas pacientes son mosaicos funcionales, lo que conlleva la aparición de manifestaciones clínicas. La IP ocurre aproximadamente en 1 de cada 40,000 a 50,000 nacimientos, con 27.6 casos nuevos al año en el mundo ${ }^{2}$. En general la enfermedad se diagnostica por las lesiones dermatológicas características y se acompaña de anomalías neurológicas y oculares.

\section{Fisiopatogenia}

En los casos de IP se han identificado mutaciones en el gen IKBKG/NEMO, que se encuentra localizado en el cromosoma $X$, específicamente en Xq28. En el 90\% de las mutaciones ocurre una deleción de los exones 4 a 10, lo que produce una pérdida completa de la función ${ }^{3}$. El gen NEMO codifica el componente regulador del complejo IKK (cinasa de I $k \mathrm{~B}$ ), el cual escinde a la $\mathrm{I} \kappa \mathrm{B}$, una proteína inhibitoria unida a $\mathrm{NF} \kappa \beta$ (nuclear factor kappa-light-chain-enhancer of activated $B$ cells) que impide que este migre al núcleo y comience la transcripción de factores que amplifican la respuesta inmunitaria, previniendo la apoptosis. La pérdida de la función de NEMO causa que IKK sea no funcional $y$, consecuentemente, la vía de $\mathrm{NF} \kappa \beta$ no pueda ser activada (Figura 1) ${ }^{3-5}$, con lo que las células mutantes se vuelven vulnerables a la apoptosis cuando son expuestas a citocinas como el factor de necrosis tumoral alfa (TNF- $\alpha$ ). Las células afectadas sobreexpresan factores quimioatrayentes, como la eotaxina, que recluta eosinófilos, y en conjunto con otros factores se genera una inflamación extensa. Este proceso afecta a las células endoteliales, las cuales, por la apoptosis, producen vaso-oclusión e isquemia de tejidos como el sistema nervioso central (SNC) y la retina ${ }^{6}$.

\section{Cuadro clínico}

La naturaleza dominante de la IP, ligada al cromosoma $X$, es causa de que afecte mayoritariamente al sexo femenino. Los varones suelen fallecer prenatalmente; los pocos casos reportados que sobreviven tienen cariotipo XXY (síndrome de Klinefelter) o mosaicismo somático, y suelen presentar características que usualmente no se asocian con IP, como inmunodeficiencia, osteopetrosis y linfedema ${ }^{7,8}$.

Todos los pacientes con la mutación presentan la enfermedad; es decir, la IP es de penetrancia completa. Las manifestaciones clínicas comprenden afecciones dermatológicas, oftálmicas y neurológicas ${ }^{9}$. Sin embargo,debido a su expresividad variable, son distintas de persona a persona.

\section{Manifestaciones cutáneas}

Los hallazgos en la piel se observan en el $100 \%$ de los pacientes por la apoptosis de las células mutadas deficientes en NEMO/IKBKG, y se distribuyen a lo largo de las líneas de Blaschko, las cuales siguen el patrón de migración de las células de la piel en la embriogénesis ${ }^{2}$. Estas líneas son normalmente invisibles, a menos que una dermatosis las evidencie. Las manifestaciones cutáneas siguen una secuencia de cuatro fases que inicia desde el nacimiento; estas pueden sobreponerse unas con otras o alguna puede no presentarse en un individuo ${ }^{10}$.

La primera fase de la enfermedad es la vesicular. Se caracteriza por la presencia de vesículas y pústulas con base eritematosa y de predominio en las extremidades (Figura 2 A). Esta fase ocurre en el $90 \%$ de los pacientes. Las lesiones se observan desde el nacimiento o en las primeras 2 semanas de vida ${ }^{11}$, y suelen desaparecer entre los 4 y 6 meses de edad. En esta fase es común encontrar eosinofilia debido a la sobreexpresión de eotaxina ${ }^{6}$. 


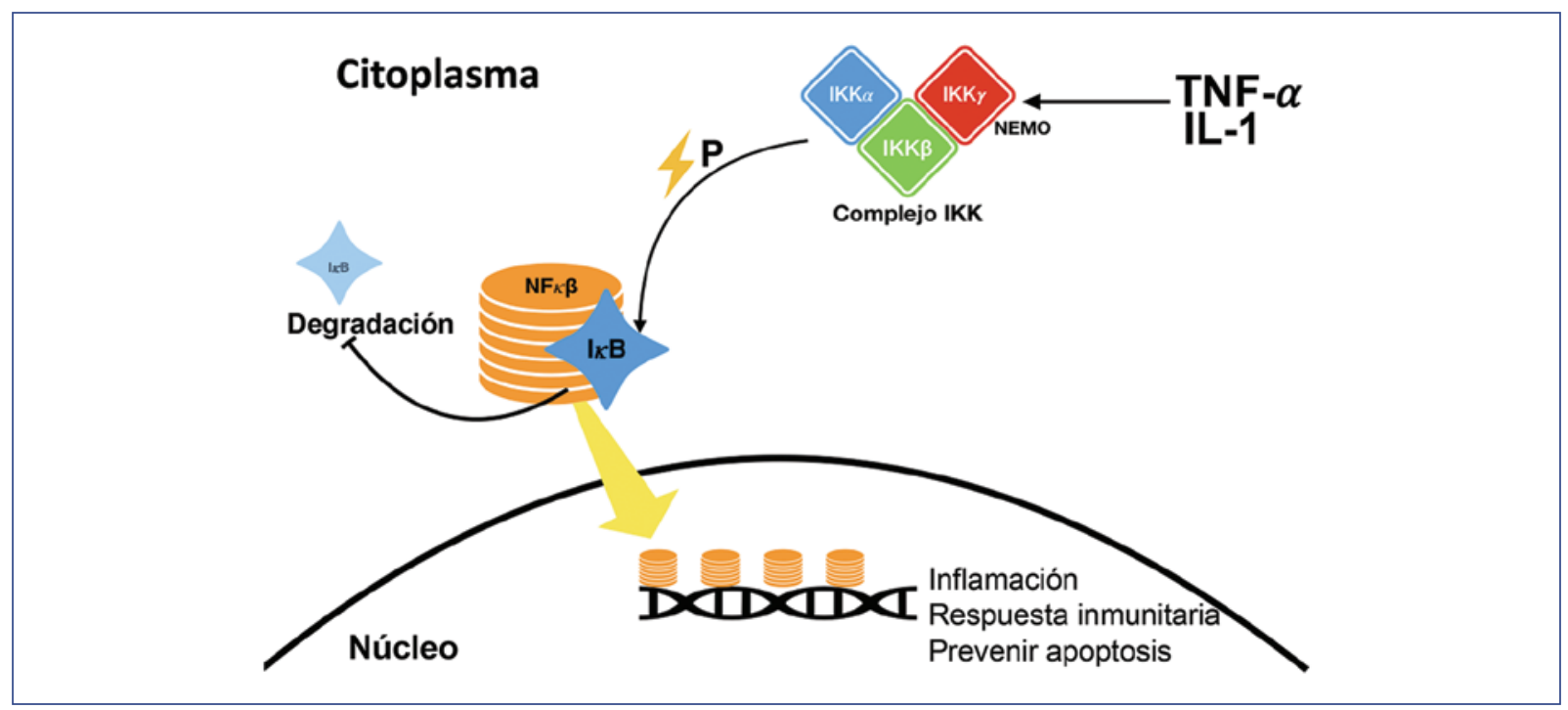

Figura 1. Activación de la vía NF $\kappa \beta$. El complejo IKK (cinasa de $I_{\kappa} B$ ) se compone de tres subunidades: IKK $\alpha$, IKK $\beta$ e IKK $\gamma$. Esta última, conocida como NEMO/IKBKG, es la proteína reguladora del complejo. Ante estímulos como el factor de necrosis tumoral alfa (TNF- $\alpha$ ) y la interleucina 1 (IL-1), este complejo escinde la proteína inhibitoria I $\kappa$ B mediante fosforilación, lo cual permite que el NFk $\beta$ (nuclear factor kappa-light-chain-enhancer of activated $B$ cells) se traslade al núcleo y se una a secuencias específicas de DNA, promoviendo la transcripción de genes relacionados con inflamación y prevención de la apoptosis.

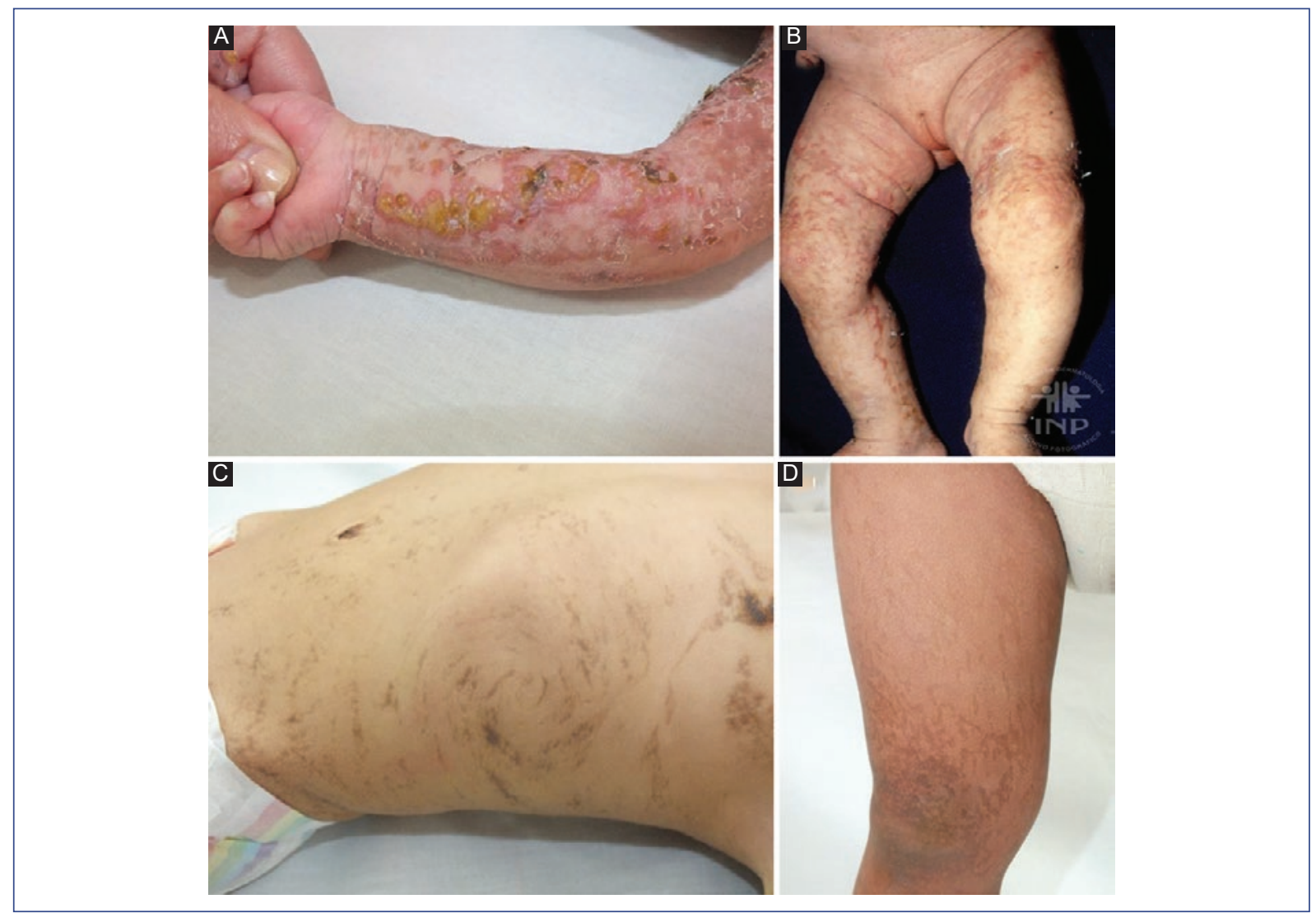

Figura 2. A: Fase vesicular. Se observan múltiples vesículas y pústulas en la extremidad superior. B: Fase verrucosa. Presencia de pápulas verrucosas con distribución lineal en las extremidades inferiores. C: Fase hiperpigmentada. Se observan las características máculas hiperpigmentadas de forma arremolinada que siguen las líneas de Blaschko. D: Fase atrófica. Se pueden apreciar placas con atrofia de distribución lineal con ausencia de pelo. 
La segunda fase, o fase verrucosa, ocurre en el $70 \%$ de los casos y se caracteriza por la presencia de pápulas de aspecto queratósico o verrucoso, que también siguen las líneas de Blaschko, pero no necesariamente con la misma distribución que las lesiones vesiculares (Figura $2 \mathrm{~B})^{2}$. Las lesiones surgen entre la segunda y la sexta semanas de vida, por lo que pueden coexistir con la primera fase y desaparecer hacia los 6 meses de edad ${ }^{2,9}$.

La tercera fase, de hiperpigmentación, es distintiva de la IP. De hecho, da su nombre a la enfermedad por el hallazgo histopatológico de una respuesta posinflamatoria que afecta la capa basal de la epidermis, causando la caída o «incontinencia» de pigmento (melanina) hacia la dermis ${ }^{10,12}$. Se observan líneas y remolinos de pigmentación de color marrón en las extremidades y el tronco que aparecen en la infancia y se desvanecen, en su mayoría, al final de la adolescencia (Figura $2 \mathrm{C})^{2}$.

La cuarta y última fase se conoce como fase de hipopigmentación o atrofia, y se caracteriza por placas lineales hipopigmentadas con ausencia de pelo e hipohidrosis (Figura 2 D). Estas placas se presentan en el $30-75 \%$ de los pacientes y predominan en las extremidades inferiores ${ }^{11}$. Usualmente se desarrollan durante la adolescencia y pueden persistir en la adultez.

\section{Manifestaciones oftálmicas}

La afección ocular en la IP, aunque no es tan prevalente, es causa de morbilidad en estos pacientes. La incidencia de las manifestaciones oscila entre el $17 \mathrm{y}$ el $25 \%$, aunque ha llegado al $77 \%$ en algunas series de casos $^{2,11}$. La afección se puede dividir en hallazgos dentro o fuera de la retina. La retina se afecta por vaso-oclusión e isquemia, lo que produce neovascularización y áreas de avascularidad periférica ${ }^{9}$. Este proceso provoca secuelas como exudados, hemorragia vítrea, fibrosis prerretiniana y desprendimiento de la retina ${ }^{9}$. Las manifestaciones no retinianas más comunes son el estrabismo y las cataratas.

\section{Manifestaciones neurológicas}

Existe compromiso neurológico en un tercio de los pacientes con IP, lo que impacta significativamente su calidad de vida. Las anomalías del SNC más comunes son crisis convulsivas (42\%), parálisis motora (26\%) y retraso cognitivo $(20 \%)^{13}$. Estas manifestaciones ocurren en el $88 \%$ de los casos antes del primer año de vida $^{13}$. Las crisis convulsivas suelen ser focales motoras. En la resonancia magnética se ha observado una

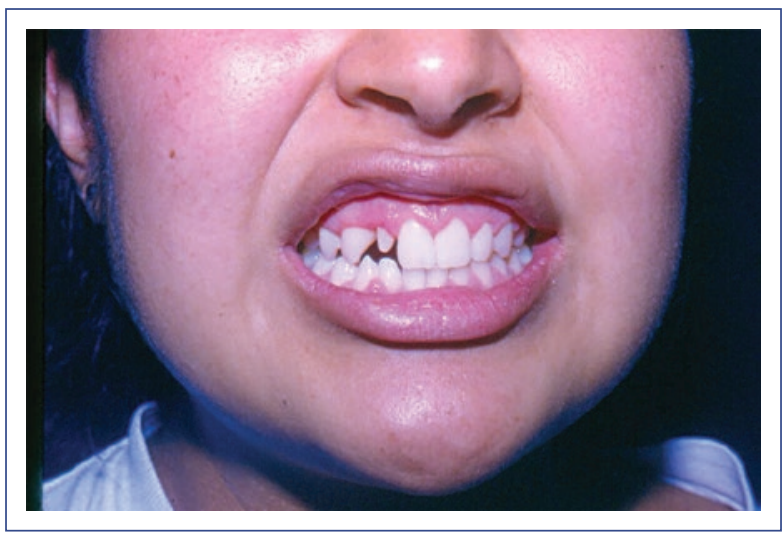

Figura 3. Dientes cónicos y microdoncia en una paciente con diagnóstico de incontinentia pigmenti.

restricción a la difusión en la sustancia blanca subcortical y áreas de hemorragia petequial ${ }^{14}$. Los estudios de imagen cerebral han demostrado alteraciones, como infartos cerebrales con zonas de necrosis, atrofia cerebral, leucomalacia periventricular, dilatación ventricular y anormalidades en el cuerpo calloso ${ }^{14,15}$, probablemente causadas por daños en la microvasculatura cerebral. La proporción de pacientes con dificultades en el aprendizaje es alta, en particular en las áreas de matemáticas $(86 \%)$ y lectura (46\%), aunque esto no está relacionado con el coeficiente intelectual, que se encuentra dentro de los rangos normales ${ }^{16}$.

\section{Otras manifestaciones}

Se ha observado afectación en el pelo en el 28-38\% de los pacientes ${ }^{2}$; la alopecia cicatricial, usualmente del vertex, es la manifestación más común. Adicionalmente, el pelo puede ser escaso en la infancia y después volverse opaco y frágil. Las alteraciones ungueales ocurren en el $40 \%$ de los pacientes y pueden afectar todas las uñas o solo una en particular ${ }^{11}$. Se ha descrito la presencia de distrofia ungueal, coiloniquia, estrías longitudinales y coloración amarillenta ${ }^{2}$. También pueden aparecer tumores queratósicos subungueales después de la pubertad, los cuales suelen ser dolorosos y pueden estar acompañados de deformidades óseas subyacentes $^{17}$. Las alteraciones dentarias se presentan en el $54-80 \%$ de los casos e incluyen anomalías de la forma (forma de cono, principalmente), hipodoncia, microdoncia y erupción retardada de los dientes (Figura 3$)^{2}$.

También se han reportado otras alteraciones en pacientes con IP. En el 1\% de los casos puede haber alteraciones mamarias, como hipoplasia y pezones 
Tabla 1. Criterios diagnósticos para incontinentia pigmenti actualizados en 2014

\begin{tabular}{|c|c|c|}
\hline Criterios mayores & Criterios menores & Condiciones para establecer el diagnóstico \\
\hline \multirow{3}{*}{$\begin{array}{l}\text { Fases cutáneas típicas } \\
\text { de la IP con distribución } \\
\text { en las líneas de } \\
\text { Blaschko: } \\
\text { - Fase vesicular } \\
\text { - Fase verrucosa } \\
\text { - Fase hiperpigmentada } \\
\text { - Fase hipopigmentada }\end{array}$} & \multirow{3}{*}{$\begin{array}{l}\text { Anomalías dentarias } \\
\text { Anomalías oculares } \\
\text { Anomalías del SNC } \\
\text { Alopecia } \\
\text { Pelo anormal (escaso, lanoso), } \\
\text { anormalidades en pestañas y cejas } \\
\text { Alteraciones ungueales } \\
\text { Alteraciones en el paladar } \\
\text { Anomalías mamarias y de pezones } \\
\text { Múltiples abortos de fetos masculinos } \\
\text { Hallazgos histopatológicos típicos en la piel }\end{array}$} & $\begin{array}{l}\text { Sin evidencia de familiar femenino de primer grado con IP: } \\
\text { - Sin evidencia de mutación, se requieren al menos dos } \\
\text { criterios mayores o uno mayor y uno menor para hacer el } \\
\text { diagnóstico } \\
\text { - Con confirmación de mutación genética se requiere un } \\
\text { criterio, ya sea mayor o menor, para el diagnóstico }\end{array}$ \\
\hline & & $\begin{array}{l}\text { Evidencia de familiar de primer grado con IP: } \\
\text { - Un criterio mayor o dos menores para el diagnóstico }\end{array}$ \\
\hline & & $\begin{array}{l}\text { En cualquier caso, la eosinofilia o la evidencia de la } \\
\text { inactivación del cromosoma } X \text { apoyan el diagnóstico }\end{array}$ \\
\hline
\end{tabular}

IP: incontinentia pigmenti; SNC: sistema nervioso central.

Adaptada de Minić, et al. ${ }^{3}$.

supernumerarios ${ }^{11}$. Adicionalmente, se han reportado pacientes con malformaciones cardiacas, como insuficiencia tricuspídea, fibrosis ventricular endomiocárdica e hipertensión pulmonar ${ }^{18}$.

\section{Diagnóstico}

En 1993, Landy y Donnai ${ }^{10}$ establecieron los criterios diagnósticos de IP, los cuales se aplicaban si el paciente tenía algún familiar de primer grado de sexo femenino con el diagnóstico de la enfermedad establecido. Si este fuera el caso, el diagnóstico se realizaría con la presencia de alguno de los siguientes criterios:

- Historia o evidencia de lesiones típicas en la piel (cualquiera de las cuatro fases).

- Placas lineales de piel atrófica y sin pelo.

- Anomalías dentales.

- Alopecia en el vertex o pelo lanoso.

- Enfermedad retiniana.

- Múltiples abortos de fetos de sexo masculino.

En caso de no tener un familiar afectado, se necesitan al menos un criterio mayor y dos criterios menores para hacer el diagnóstico:

- Criterios mayores:

- Erupción neonatal típica.

- Hiperpigmentación lineal típica.

- Lesiones lineales, atróficas y alopécicas.

- Criterios menores:

- Alteraciones dentales.

- Alopecia o pelo lanoso.

- Alteraciones ungueales.

- Enfermedad retiniana.

En el año 2014, estos criterios fueron actualizados por Minić, et al. ${ }^{3}$, quienes consideraron la presencia de cualquiera de las fases cutáneas como criterios mayores. En los criterios menores se agregaron anormalidades del SNC, hallazgos oculares extrarretinianos, histopatología típica en la piel y alteraciones mamarias y en los pezones (Tabla 1).

\section{Diagnóstico diferencial}

Las dermatosis que deben incluirse en el diagnóstico diferencial van de acuerdo con la fase de la IP en la que se evidencien ${ }^{3}$. En la primera fase se consideran dermatosis con presencia de vesículas y ampollas, como el impétigo ampolloso, la epidermólisis ampollosa y la infección por virus herpes simple. En la segunda fase se debe sospechar de verrugas vulgares, nevos epidérmicos y el síndrome NEVIL (nevo epidérmico verrugoso inflamatorio lineal). La tercera fase puede asemejarse a un mosaicismo pigmentario. En la cuarta fase se debe pensar en las diferentes displasias ectodérmicas.

\section{Manejo}

Las lesiones en la piel no requieren tratamiento específico, pues se resuelven espontáneamente. Se recomienda seguir las medidas generales de la piel y aplicar emolientes. Los antibióticos tópicos o sistémicos no deben usarse de manera sistemática, sino que deben reservarse para casos con signos de infección secundaria.

Con respecto a las manifestaciones neurológicas, no existe tratamiento específico. Las crisis convulsivas se deben manejar de manera sintomática. Existe el reporte de un caso con crisis convulsivas refractarias a anticonvulsivantes que mostró mejoría con el uso de corticosteroides a altas dosis, lo que apoya la teoría de que la 
inflamación es parte importante de la fisiopatología ${ }^{19}$. Se recomienda realizar una resonancia magnética basal en el periodo neonatal o en el momento del diagnóstico, la cual debe repetirse en caso de crisis convulsivas u otros signos neurológicos ${ }^{2,20}$. Se debe realizar una exploración oftalmológica con fondo de ojo en el momento del diagnóstico y se recomienda dar seguimiento oftalmológico mensualmente durante los primeros 4 meses de vida, trimestralmente del cuarto al duodécimo mes, semestralmente hasta los 3 años y anualmente de por vida². El tratamiento de fotocoagulación con láser en áreas con neovascularización puede indicarse para prevenir el desprendimiento de la retina. Existen reportes de casos en los que se usaron inhibidores del factor de crecimiento endotelial vascular, como bevacizumab y ranibizumab, en conjunto con el láser, con resultados positivos ${ }^{21,22}$.

Las pacientes con IP son fértiles, por lo que deben recibir asesoramiento genético. En cada embarazo, existe el $50 \%$ de probabilidad de heredar la mutación. Sin embargo, dado que la condición es letal en los hombres, existe el $33 \%$ de probabilidad de que nazca una niña no afectada, el 33\% para un niño no afectado y el $33 \%$ para una niña afectada con la enfermedad en los embarazos que llegan a término².

\section{Pronóstico}

Los pacientes con IP sin compromiso oftalmológico o neurológico clínicamente significativo cuentan con un pronóstico bueno y una esperanza de vida normal. Sin embargo, las anomalías en la piel y los anexos son permanentes, por lo que pueden ser un motivo de preocupación para los pacientes. En los casos que cursan con afectaciones oftalmológicas y neurológicas, el curso clínico es variable, dependiendo de la gravedad de las manifestaciones.

La IP es una genodermatosis dominante ligada al cromosoma $X$ con manifestaciones cutáneas características que ayudan al diagnóstico adecuado y al inicio oportuno del manejo de las complicaciones neurológicas y oculares. Para las enfermedades raras, como la IP, el bajo número de casos no solo dificulta el diagnóstico, sino también la implementación de protocolos de tratamiento y el diseño de fármacos dirigidos a la fisiopatología del padecimiento. Las áreas de investigación contemplan, a futuro, la terapia génica dirigida con dianas terapéuticas, como los inhibidores de NF $\kappa \beta$, que podrían ser aplicados para prevenir la afección neurológica y retiniana9. Por último, es importante recalcar que el manejo multidisciplinario, incluyendo los servicios de dermatología, neurología, oftalmología y genética, es fundamental para dar un correcto manejo a los pacientes con IP.

\section{Responsabilidades éticas}

Protección de personas y animales. Los autores declaran que para esta investigación no se han realizado experimentos en seres humanos ni en animales.

Confidencialidad de los datos. Los autores declaran que han seguido los protocolos de su centro de trabajo sobre la publicación de datos de pacientes.

Derecho a la privacidad y consentimiento informado. Los autores han obtenido el consentimiento informado de los pacientes y sujetos referidos en el artículo. Este documento obra en poder del autor de correspondencia.

\section{Conflicto de intereses}

Los autores declaran no tener ningún conflicto de intereses.

\section{Financiamiento}

Ninguno.

\section{Bibliografía}

1. Smahi A, Courtois G, Rabia SH, Döffinger R, Bodemer C, Munnich A, et al. The NF-kappaB signalling pathway in human diseases: from incontinentia pigmenti to ectodermal dysplasias and immune-deficiency syndromes. Hum Mol Genet. 2002;11:2371-5.

2. Greene-Roethke $C$. Incontinentia pigmenti: a summary review of this rare ectodermal dysplasia with neurologic manifestations, including treatment protocols. J Pediatr Health Care. 2017;31:e45-52.

3. Minić S, Trpinac D, Obradović M. Incontinentia pigmenti diagnostic criteria update. Clin Genet. 2014;85:536-42.

4. Smahi A, Courtois G, Vabres P, Yamaoka S, Heuertz S, Munnich A, et al. Genomic rearrangement in NEMO impairs NF-kappaB activation and is a cause of incontinentia pigmenti. The International Incontinentia Pigmenti (IP) Consortium. Nature. 2000;405:466-72.

5. Nelson DL. NEMO, NFkappaB signaling and incontinentia pigmenti. Curr Opin Genet Dev. 2006;16:282-8.

6. Jean-Baptiste S, O'Toole EA, Chen M, Guitart J, Paller A, Chan LS. Expression of eotaxin, an eosinophil-selective chemokine, parallels eosinophil accumulation in the vesiculobullous stage of incontinentia pigmenti. Clin Exp Immunol. 2002;127:470-8.

7. Kenwrick S, Woffendin H, Jakins T, Shuttleworth SG, Mayer E, Greenhalgh $\mathrm{L}$, et al. Survival of male patients with incontinentia pigmenti carrying a lethal mutation can be explained by somatic mosaicism or Klinefelter syndrome. Am J Hum Genet. 2001;69:1210-7.

8. Fusco F, Fimiani G, Tadini G, Michele D, Ursini MV. Clinical diagnosis of incontinentia pigmenti in a cohort of male patients. J Am Acad Dermatol. 2007;56:264-7.

9. Swinney CC, Han DP, Karth PA. Incontinentia pigmenti: a comprehensive review and update. Ophthalmic Surg Lasers Imaging Retina. 2015;46:650-7.

10. Landy SJ, Donnai D. Incontinentia pigmenti (Bloch-Sulzberger syndrome). J Med Genet. 1993;30:53-9.

11. Cláudia SP, Júlia KR, Luana B, Fernanda D, Carolina WC, Giovanni MT, et al. Incontinentia pigmenti. An Bras Dermatol. 2014;89:26-36.

12. Sáez-de-Ocariz M, Orozco-Covarrubias L, Duran-McKinster C, Palacios-López C, Ruiz-Maldonado R. Incontinencia pigmenti: estado actual y experiencia en el Instituto Nacional de Pediatría de México. Dermatol Pediatr Lat. 2006;4:101-10. 
13. Minić S, Trpinac D, Obradović M. Systematic review of central nervous system anomalies in incontinentia pigmenti. Orphanet J Rare Dis. 2013;8:25.

14. Narayanan MJ, Rangasamy S, Narayanan V. Incontinentia pigmenti (Bloch-Sulzberger syndrome). Handb Clin Neurol. 2015:132:271-80.

15. Meuwissen ME, Mancini GM. Neurological findings in incontinentia pigmenti; a review. Eur J Med Genet. 2012;55:323-31.

16. Pizzamiglio MR, Piccardi L, Bianchini F, Canzano L, Palermo L, Fusco F, et al. Incontinentia pigmenti: learning disabilities are a fundamental hallmark of the disease. PLoS One. 2014;9:e87771.

17. Montes CM, Maize JC, Guerry-Force ML. Incontinentia pigmenti with painful subungual tumors: a two-generation study. J Am Acad Dermatol. 2004;50:S45-52.

18. Onnis G, Diociaiuti A, Zangari $P$, D'Argenio $P$, Cancrini $C$, lughetti $L$, et al. Cardiopulmonary anomalies in incontinentia pigmenti patients. Int J Dermatol. 2018;57:40-5.
19. Wolf DS, Golden WC, Hoover-Fong J, Applegate C, Cohen BA, Germain-Lee EL, et al. High-dose glucocorticoid therapy in the management of seizures in neonatal incontinentia pigmenti: a case report. J Child Neurol. 2015;30:100-6.

20. Poretti A, Northington FJ. Brain injury in neonatal incontinentia pigmenti: the role of multimodality neuroimaging. Dev Med Child Neurol. 2016; 58:1000-1.

21. Ho M, Yip WWK, Chan VCK, Young AL. Successful treatment of refractory proliferative retinopathy of incontinentia pigmenti by intravitreal ranibizumab as adjunct therapy in a 4-year-old child. Retin Cases Brief Rep. 2017;11:352-5.

22. Shah PK, Bachu S, Narendran V, Kalpana N, David J, Srinivas CR. Intravitreal bevacizumab for incontinentia pigmenti. J Pediatr Ophthalmo Strabismus. 2013;50:e52-4. 